\title{
Pityriasis folliculorum colocalized with vitiligo: An example of immunocompromised cutaneous district
}

\section{Ayse Tulin Mansur}

\author{
Dermatology Department, Baskent University Istanbul Hospital, Istanbul, Turkey \\ Corresponding author: Dr. Ayse Tulin Mansur, E-mail: tulinmansur@gmail.com
}

\begin{abstract}
The concept of immunocompromised cutaneous district suggests that different types of skin injuries can render the affected areas vulnerable to subsequent opportunistic infections, tumors, and immune reactions. Herein a 44-year-old woman with facial vitiligo associated with pityriasis folliculorum, a type of demodicosis, is reported. The right cheek with a large patch of vitiligo showed clinical, dermoscopic and microscopic evidents of demodicosis, while the other facial areas without vitiligo were devoid of Demodex mites. This case is another example of immunocompromised cutaneous district, probably developed due to immunologic alterations and increased ultraviolet light exposure caused by vitiliginous skin.
\end{abstract}

Key words: Demodex; Demodicosis; Pityriasis folliculorum; Rosacea; Vitiligo

\section{INTRODUCTION}

Pityriasis folliculorum $(\mathrm{PF})$ is characterized by hyperkeratotic follicles filled with Demodex mites, which results tiny facial spicules [1,2]. Vitiligo is an acquired depigmentation disorder caused by complex influences on melanocytes, including genetics, autoimmunity, cytotoxic and oxidant-antioxidant mechanisms [3]. Herein a case of PF colocalized with facial vitiligo is described and the probable mechanisms of this association are discussed.

\section{CASE REPORT}

A 44-year-old woman complained of a milky white patch, dry skin and redness on the right cheek, present for 1 year. During the last 6 months white spots had developed also on the genital area and around the eyes. She had been suffering from panic attack disorder since 2009, and had treated with venlafaxine and olanzapin. Dermatological examination showed bilateral depigmented patches involving periorbital and malar regions, in addition to a large depigmented and erythematous patch on the right cheek. In this latter area follicular minute spicules were evident, causing a sandpaper feeling on palpation (Fig. 1) Examination under Wood lamp revealed accentuation of whitening over depigmented areas confirming the diagnosis of vitiligo (Figs. 2a and 2b). On the left cheek no depigmentation, erythema, or follicular spicules were noted, besides no accentuation with Wood light (Figs. 3a and 3b). Dermoscopy showed mites protruding from follicular orifices (Fig. 2c). Standardized skin surface biopsies taken from the areas showing PF revealed ample amount of Demodex mites, either as follicular bunchs of 4-7 mites, or freely floating isolated mites (Figs. 2d and 2e).

Laboratory investigations including complete blood cell count, TSH, anti-TPO, serum vitamin B12 level, and fasting glucose were within normal limits.

\section{DISCUSSION}

Demodex mites (D. folliculorum and D. brevis) are common commensals of the skin, typically found on the face. They are usually resident in the pilosebaceous unit at a density of $\leq 5 \mathrm{~cm}^{2}$. An increase in the number

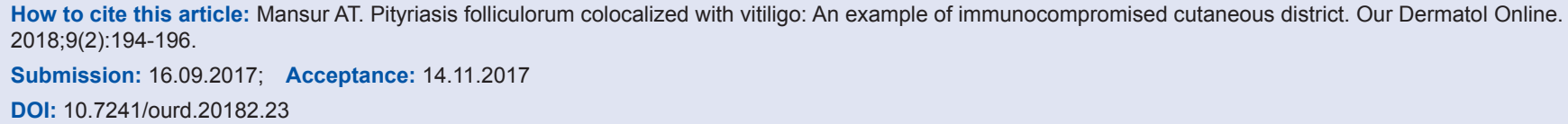




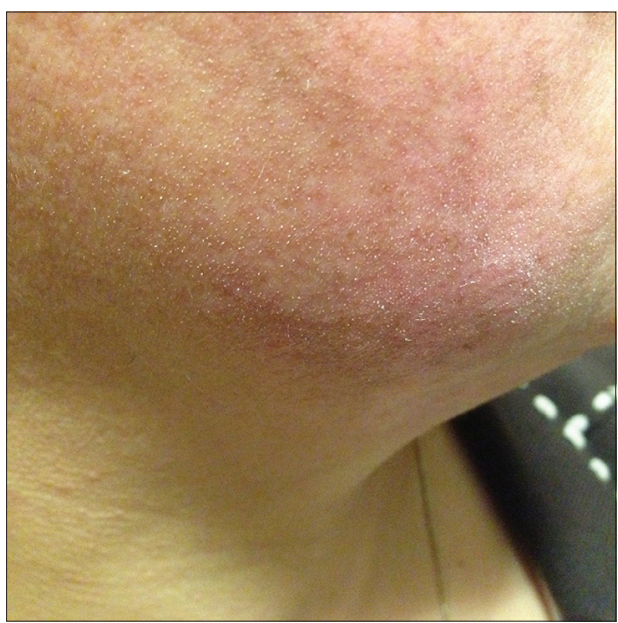

Figure 1: Follicular spicules and erythema overlying the depigmented patches

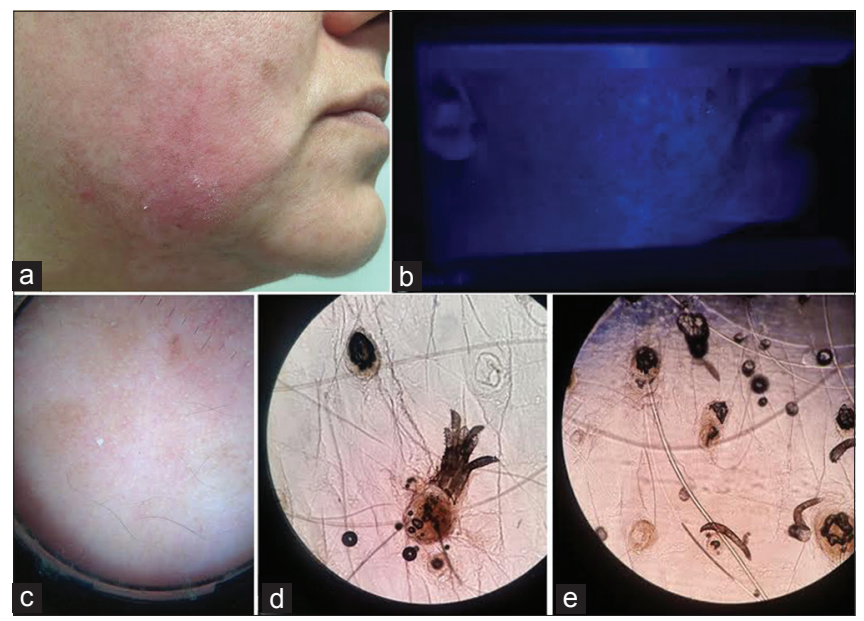

Figure 2: $(a, b)$ White patches showing bright, blue-white fluorescence with sharp borders under Wood light; (c,d,e): Dermoscopy of the lesional area showing Demodex mites protruding from the follicular orifices. Superficial skin biopsy revealing profuse amount of Demodex mites.

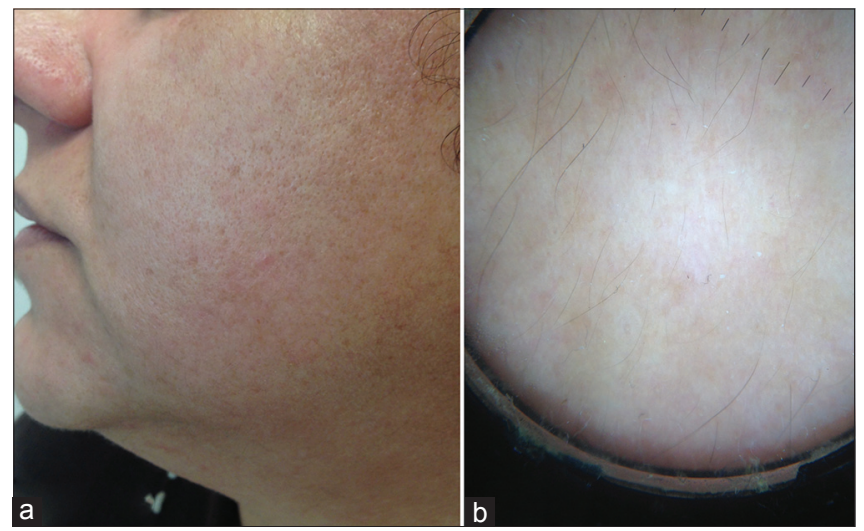

Figure 3: $(a, b)$ Left cheek free from vitiligo and PF

of mites in the pilosebaceous unit, or less commonly, penetration of mites into the dermis is considered pathogenic [1]. Demodicosis is a term refers to chronic skin disorders induced by Demodex spp., including pustular folliculitis, PF, rosacea-like demodicosis, and demodicosis gravis [2].

In $\mathrm{PF}$ the mites proliferate profusely with no, or a low immune response from the host. They plug the hair follicles and cause erythema and a prominent, sandpaper-like dryness. Tiny spicules composed of mite bunches are seen with naked eye and are very characteristic for this entity [2].

As few individuals develop symptoms in contrast to high prevalance of mites in general population, demodicosis can be considered as a multifactorial disorder, induced by external and internal factors. Immunosuppression may play a role on transition from an asymptomatic infestation to the development of clinical features. Demodex density has been reported to be high in immunosuppressive conditions such as HIV infection, chronic renal disease, diabetes mellitus, malnutrition, leukemia, cancer, corticosteroid or cytostatic therapy $[2,4,5]$.

The presented patient had no history of systemic or local immunosuppresive treatment, including topical calcineurin inhibitors and corticosteroids. Her past medical history was unremarkable except vitiligo, emotional stress and psychiatric problems.

Vitiligo colocalized with PF has not been reported previously. This concurrence seems to be more than a simple coincidence, as the areas of the left cheek lacking vitiligo lesions were devoid of mites either.

The exact mechanisms of this colocalization are not clear yet. There is plenty of evidence for altered immunological processes in vitiligo, involving both innate and adaptive immune system. Oxidant stress and inadequate antioxidative defences leading to accumulation of reactive oxygen species also play important roles in the development of vitiligo $[3,6]$. It has long been known that ultraviolet (UV) radiation, in particular the UVB range, suppresses the immune system in several ways [7]. Loss of melanocytes in vitiligo increases the detrimental effects of UV. On the other hand, exposure to UV is an external stimuli that may trigger rosacea lesions [8]. In our patient increased UV exposure and insufficient defences due to vitiligo may have augmented the negative effects of UV on Demodex control [3,6]. Furthermore, an old hypothesis "locus minoris resistentiae (LMR)" has recently gained a new popularity by the concept of 
immunocompromised cutaneous district proposed by Ruocco et al. It suggests that different types of skin injuries including UV radiation, can render the affected areas vulnerable to subsequent opportunistic infections, tumors, and immune reactions, due to a local immune imbalance [9].

\section{CONCLUSION}

In this context, vitiligo may be considered a LMR due to its susceptibility to UV damages, and immunologic alterations, leading to increased proliferation of mites.

\section{REFERENCES}

1. Rather PA, Hassan I. Human demodex mite: the versatile mite of dermatological importance. Indian J Dermatol. 2014;59:60-6.

2. Hsu CK, Hsu MM, Lee JY. Demodicosis: a clinicopathological study. J Am Acad Dermatol. 2009;60:453-62.
3. Laddha NC, Dwivedi M, Mansuri MS, Gani AR, Ansarullah M, Ramachandran AV, et al. Vitiligo: interplay between oxidative stress and immune system. Exp Dermatol. 2013;22:245-50.

4. Elston DM. Demodex mites: facts and controversies. Clin Dermatol. 2010;28:502-4.

5. Kaya S, Selimoglu MA, Kaya OA, Ozgen U. Prevalence of Demodex folliculorum and Demodex brevis in childhood malnutrition and malignancy. Pediatr Int. 2013;55:85-9.

6. Manga P, Elbuluk N, Orlow SJ. Recent advances in understanding vitiligo. F1000Res. 2016;5 pii: F1000 Faculty Rev-2234.

7. Schwarz T The Many Faces of UVR-Induced Immunosuppression. J Investig Dermatol Symp Proc. 2015;17:22-3.

8. Margalit A, Kowalczyk MJ, Żaba R, Kavanagh K. The role of altered cutaneous immune responses in the induction and persistence of rosacea. J Dermatol Sci. 2016;82:3-8.

9. Piccolo V, Baroni A, Russo T, Schwartz RA. Ruocco's immunocompromised cutaneous district. Int J Dermatol. 2016;55:135-41.

Copyright by Ayse Tulin Mansur. This is an open access article distributed under the terms of the Creative Commons Attribution License, which permits unrestricted use, distribution, and reproduction in any medium, provided the original author and source are credited.

Source of Support: Nil, Conflict of Interest: None declared. 